\title{
A Simple and Effective Synthesis of 3- and 4-((Phenylcarbamoyl)oxy)benzoic Acids
}

\author{
Urban Košak $^{1}$ and Stanislav Gobec ${ }^{1, *}$ \\ ${ }^{1}$ Faculty of Pharmacy, University of Ljubljana, Aškerčeva 7, 1000 Ljubljana, Slovenia \\ * Corresponding author: E-mail: stanislav.gobec@ffa.uni-lj.si \\ Tel.: + 386 (1)4769585; fax: + 386 (1)4258031
}

Received: 03-23-2020

\begin{abstract}
Phenserine, posiphen, tolserine and cymserine and its derivatives are experimental Alzheimer's disease drugs that contain a phenyl phenylcarbamate moiety that is responsible for their anti-Alzheimer activities. We have developed a simple (3 steps) and effective (overall yields 76-90\%) method for preparing 3- and 4-((phenylcarbamoyl)oxy)benzoic acids which can be reacted with amines to produce phenyl phenylcarbamate moiety containing amides as new potential anti-Alzheimer disease drugs. The synthesized carboxylic acids are thus important building blocks with potential use in medicinal chemistry and drug discovery.
\end{abstract}

Keywords: ((Phenylcarbamoyl)oxy)benzoic acids; phenyl isocyanates; carbamates; building blocks; Alzheimer's disease.

\section{Introduction}

Alzheimer's disease (AD) is a progressive neurodegenerative brain disorder. ${ }^{1}$ The synaptic dysfunction and neurodegeneration in $\mathrm{AD}$ most severely affects the cholinergic system. ${ }^{2}$ This decreases the levels of the neurotransmitter acetylcholine $(\mathrm{ACh}),{ }^{3}$ which then produces cognitive impairment and memory loss, ${ }^{4}$ characteristic for patients with AD. Several compounds are currently being evaluated in preclinical and clinical trials for efficacy in $\mathrm{AD}$, including cholinesterase (ChE) inhibitors which increase the levels of $\mathrm{ACh}$ in the brain: phenserine, posiphene, tolserine and cymserine and its derivatives (Figure 1). ${ }^{5}$ These experimental Alzheimer's disease drugs all contain the phenyl phenylcarbamate moiety or its derivative. Phenserine ${ }^{6}$ and posiphen ${ }^{7}$ contain a phenyl phenylcarbamate moiety, tolserine ${ }^{8}$ contains a phenyl ortho-tolylcarbamate moiety and cymserine and its derivatives ${ }^{9,10}$ contain a phenyl (4-isopropylphenyl)carbamate moiety (Figure 1).

Phenserine, posiphen, tolserine and cymserine and its derivatives are pseudo-irreversible carbamate inhibitors of ChEs where the phenyl phenylcarbamate moiety is responsible for their biological activity. Their mechanism of inhibition involves a rapid initial covalent reaction between their carbamate carbonyl group and the catalytic serine in the active site of ChEs (carbamoylation). The inhibited (carbamoylated) $\mathrm{ChE}$ is then reactivated by a slow hydrolysis (decarbamylation) of the active enzyme serine (Scheme 1). ${ }^{11,12}$

As part of our development of new ChE inhibitors as potential anti-Alzheimer disease drugs, we designed compounds with the general formula $\mathbf{1}$ that contain the phenyl phenylcarbamate moiety (Scheme 2A). These compounds were designed based on the structures of our previously reported ChE inhibitors. ${ }^{13-15}$ We planned to synthesize compounds with the general formula $\mathbf{1}$ by utilizing one of several methods for the synthesis of carbamates, ${ }^{16,17}$ i.e. reacting phenols with the general formula 2 with various phenyl isocyanates (3) in the presence of a catalytic amount of 4-dimethylamino pyridine (4-DMAP) in $\mathrm{CH}_{2} \mathrm{Cl}_{2}$ or DMF (Scheme 2A). ${ }^{18,19}$ However, this reaction did not produce the desired carbamates as no reaction was observed. Therefore, we had to plan an alternative synthetic route. We decided to use 3- and 4-((phenylcarbamoyl)oxy) benzoic acids (4) and react them with various amines (5) which we have previously used to synthesize amide ${ }^{13,14}$ and sulfonamide ${ }^{14,15} \mathrm{ChE}$ inhibitors, in the presence of coupling reagent TBTU and $\mathrm{N}, \mathrm{N}$-diisopropylethylamine (DIPEA) in $\mathrm{CH}_{2} \mathrm{Cl}_{2}{ }^{20}$ to produce the designed amides (Scheme 2B).

The problem was that 3- and 4-((phenylcarbamoyl) oxy)benzoic acids (4; Scheme 2B) are not commercially available and procedures for their preparation have also not been reported yet. Herein we describe how we solved 
<smiles>CN1CC[C@@]2(C)c3cc(OC(=O)Nc4ccccc4)ccc3N(C)[C@H]12</smiles>

Phenserine<smiles>CN1CC[C@@]2(C)c3cc(OC(=O)Nc4ccccc4)ccc3N(C)[C@H]12</smiles>

Posiphen<smiles>Cc1ccccc1NC(=O)Oc1ccc2c(c1)[C@@]1(C)CCN(C)[C@H]1N2C</smiles>

Tolserine<smiles>CC(C)c1ccc(NC(=O)Oc2ccc3c(c2)[C@@]2(C)CCN(C)[C@H]2N3C)cc1</smiles>

Cymserine<smiles>CC(C)c1ccc(NC(=O)Oc2ccc3c(c2)C2(C)CCNC32)cc1</smiles>

Bisnorcymserine

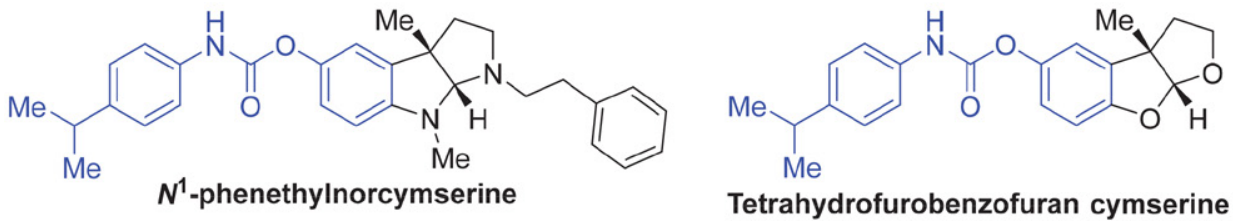<smiles>CC(C)c1ccc(NC(=O)Oc2ccc3c(c2)[C@@]2(C)CO[C@@H](C3)O2)cc1</smiles>

Dihydrobenzodioxepine cymserine

Figure 1. Structures of phenyl phenylcarbamate containing experimental Alzheimer's disease drugs.

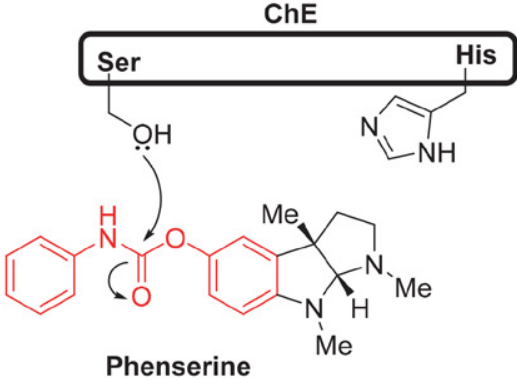

Phenserine

ChE

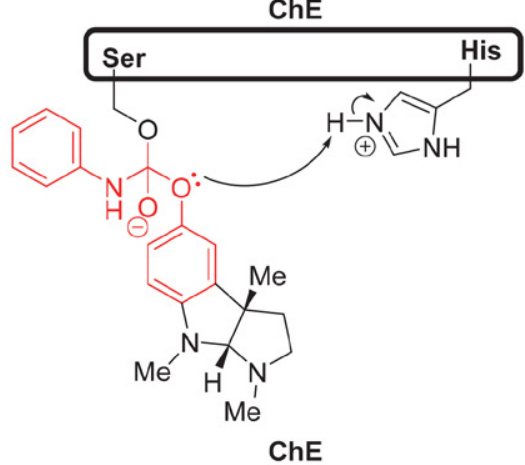

ChE

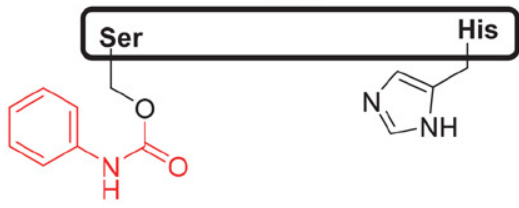

ChE inhibited by carbamoylation
ChE

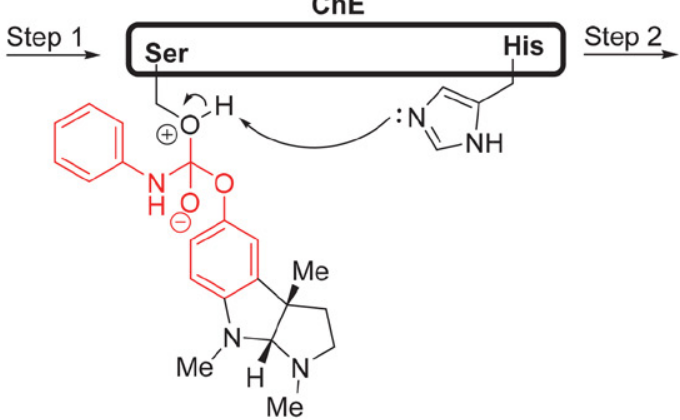

ChE

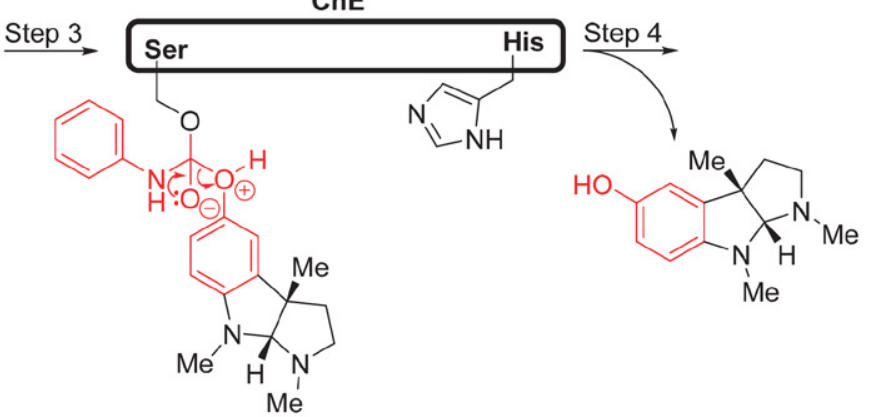

Scheme 1. Mechanism of ChE inhibition by phenserine. 
a)

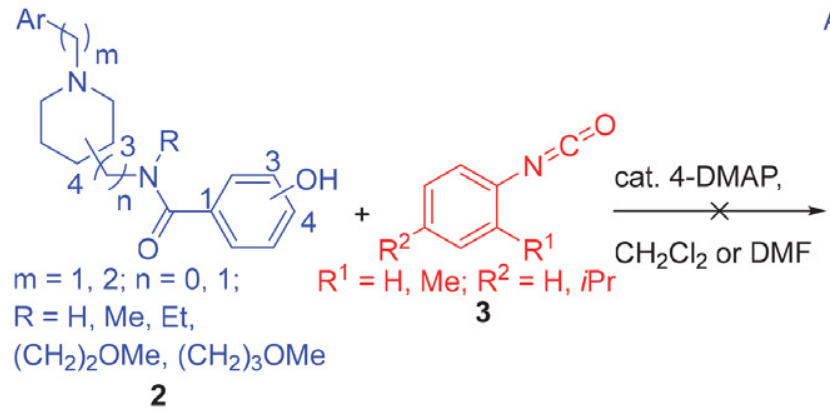

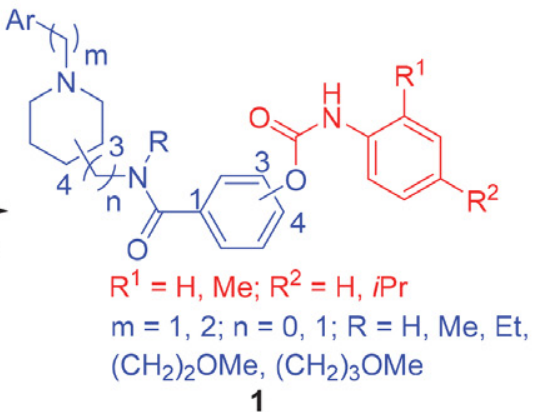

b)

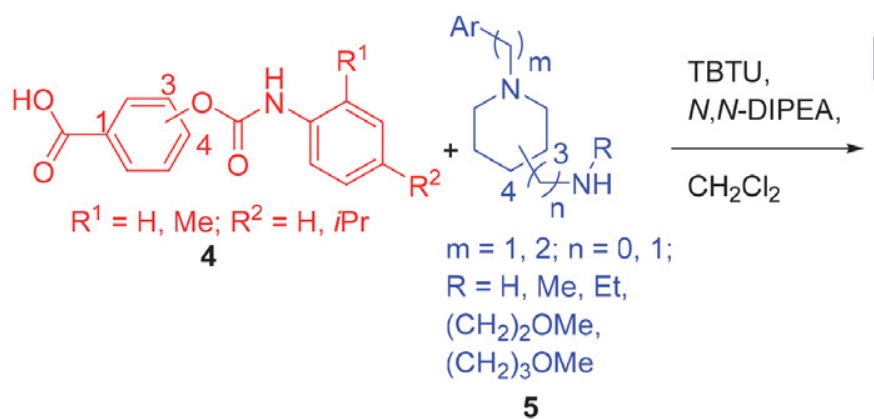<smiles>C1=C[Al]C[Al]1</smiles>

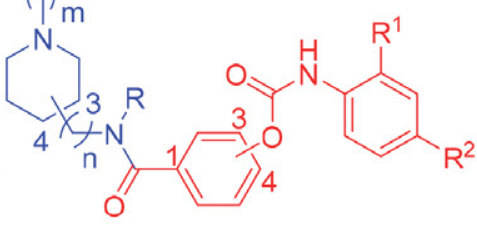

$\mathrm{R}^{1}=\mathrm{H}, \mathrm{Me} ; \mathrm{R}^{2}=\mathrm{H}, \mathrm{Pr}$ $m=1,2 ; n=0,1 ; R=H, M e, E t$, $\left(\mathrm{CH}_{2}\right)_{2} \mathrm{OMe},\left(\mathrm{CH}_{2}\right)_{3} \mathrm{OMe}$

1

Scheme 2. Synthesis of new ChE inhibitors as potential anti-Alzheimer disease drugs with the general formula $\mathbf{1}$.

this problem by developing a simple procedure to produce these building blocks in high overall yields.

\section{Experimental}

\section{1. General Chemistry Methods}

${ }^{1} \mathrm{H}$ NMR and ${ }^{13} \mathrm{C}$ NMR were recorded at 400.130 $\mathrm{MHz}$ and $100.613 \mathrm{MHz}$, respectively, on an NMR spectrophotometer (Bruker Avance III). The chemical shifts $(\delta)$ are reported in parts per million ( $\mathrm{ppm}$ ) and are referenced to the deuterated solvent used. The coupling constants $(J)$ are reported in $\mathrm{Hz}$, and the splitting patterns are indicated as: s, singlet; br. s, broad singlet; d, doublet; dd, doublet of doublets; td, triplet of doublets; $h$, hextet; $m$, multiplet; $t$, triplet; br. t, broad triplet; $\mathrm{dt}$, doublet of triplets; $\mathrm{tt}$, triplet of triplets; q, quartet; qd, quartet of doublets. Infrared (IR) spectra were recorded on a FT-IR spectrometer (System Spectrum BX; Perkin-Elmer). ATR IR spectra were recorded on a FT-IR spectrometer (Thermo Nicolet Nexus 470 ESP). Micro-analyses were performed on a Perkin-Elmer C, H, N Analyzer $240 \mathrm{C}$. The analyses are indicated by the symbols of the elements and they were within $\pm 0.4 \%$ of the theoretical values. Mass spectra were recorded on a LC-MS/MS system (Q Executive Plus; Thermo Scientific, MA, USA). Melting points were determined on a Leica hot-stage microscope and are uncorrected. Evaporation of the solvents was performed under reduced pressure. Reagents and solvents were purchased from Acros Organics, Alfa Aesar, Euriso-Top, Fluka, Merck, Sigma-Aldrich, and TCI Europe, and were used without further purification, unless otherwise stated. Flash column chromatography was performed on silica gel 60 for column chromatography (particle size, 230-400 mesh). Analytical thin-layer chromatography was performed on silica gel aluminum sheets $(0.20 \mathrm{~mm}$; 60 F254; Merck), with visualization using ultraviolet light and/or visualization reagents. Analytical reversed-phase UPLC method A was performed on an LC system (Dionex Ultimate 3000 Binary Rapid Separation; Thermo Scientific) equipped with an autosampler, a binary pump system, a photodiode array detector, a thermostated column compartment, and the Chromeleon Chromatography Data System. The detector on UPLC system was set to $210 \mathrm{~nm}$ and $254 \mathrm{~nm}$. The column used for method A was a C18 analytical column $(50 \times 2.1 \mathrm{~mm}$, $1.8 \mu \mathrm{m}$; Acquity UPLC HSS C18SB). The column was thermostated at $40^{\circ} \mathrm{C}$.

Method $A$ : The sample solution $(1 \mu \mathrm{L} ; 0.2 \mathrm{mg} / \mathrm{mL}$ in $\mathrm{MeCN}$ ) was injected and eluted at a flow rate of $0.4 \mathrm{~mL} /$ min, using a linear gradient of mobile phase $\mathrm{A}(\mathrm{MeCN})$ and mobile phase $\mathrm{B}(0.1 \%[\mathrm{v} / \mathrm{v}]$ aqueous TFA). The gradient for method A (for mobile phase A) was: $0-2 \mathrm{~min}, 20 \%$; 2-5 $\min , 20-90 \%$; 5-8 $\min , 90 \%$.

\section{2. General Synthetic Procedures}

\section{2. 1. General Procedure for Synthesis of Benzyl Esters 6 and 8 (General Procedure 1)}

To a $100-\mathrm{mL}$ round-bottom flask equipped with a stirring bar, hydroxybenzoic acid $(5.000 \mathrm{~g}, 36.177 \mathrm{mmol}$, 1.0 mol. equiv.) and DMF (50 mL) were added. The resulting solution was stirred and $\mathrm{Na}_{2} \mathrm{CO}_{3}(3.837 \mathrm{~g}, 36.177$ 
mmol, 1.0 mol. equiv.) was added. Benzyl bromide (4.297 $\mathrm{mL}, 36.177 \mathrm{mmol}, 1.0 \mathrm{~mol}$. equiv) was added dropwise to the suspension and the reaction mixture was stirred for 24 hours at room temperature, then poured into a $500-\mathrm{mL}$ separating funnel. Water $(100 \mathrm{~mL})$ was added and the mixture was extracted with $\mathrm{Et}_{2} \mathrm{O}(3 \times 150 \mathrm{~mL})$. The combined organic phases where transferred into a 1-L separating funnel, washed with water $(3 \times 450 \mathrm{~mL})$ followed by sat. brine solution $(450 \mathrm{~mL})$, dried over anhyd. $\mathrm{Na}_{2} \mathrm{SO}_{4}$, and evaporated to produce the benzyl hydroxybenzoate as a colourless oil which solidified into a white solid after cooling. This product was used in the next step without further purification.

\section{2. 2. General Procedure for Synthesis of Carbamates 10-15 (General Procedure 2)}

To a round-bottom flask equipped with a stirring bar, benzyl hydroxybenzoate (1.0 mol. equiv.) and $\mathrm{CH}_{2} \mathrm{Cl}_{2}$ $(c=0.3 \mathrm{M})$ were added. The resulting solution was stirred and 4-DMAP (0.01 mol. equiv.) was added. Phenyl isocyanate, 2-methylphenyl isocyanate or 4-isopropylphenyl isocyanate ( 1.0 mol. equiv.) was added dropwise and the reaction mixture was stirred for 24 hours at room temperature, then evaporated to produce the carbamates. These products were used in the next step without further purification.

\section{2. 3. General Procedure for Debenzylation of Benzyl Esters Yielding 16-21 (General Procedure 3)}

To a round-bottom flask equipped with a stirring bar, benzyl ester (1.0 mol. equiv.) and inhibitor-free THF $(c=0.02 \mathrm{~g} / \mathrm{mL})$ were added. The resulting solution was stirred and agitated with a stream of argon for $30 \mathrm{~min} .10 \%$ $\mathrm{Pd} / \mathrm{C}$ (5\% mass of benzyl ester) was added and the resulting suspension was agitated with a stream of hydrogen for $30 \mathrm{~min}$. The reaction mixture was stirred under an atmosphere of hydrogen for 24 hours then agitated with a stream of argon for $30 \mathrm{~min}$, filtered with suction through a pad of Celite and evaporated to produce the carboxylic acid.

\section{3. Synthesis and Characterization of Compounds}

\section{3. 1. Synthesis of Benzyl 3-Hydroxybenzoate (6)}

Synthesized from 3-hydroxybenzoic acid (7) $(5.000$ g, $36.177 \mathrm{mmol}, 1.0$ mol. equiv.), $\mathrm{Na}_{2} \mathrm{CO}_{3}(3.837 \mathrm{~g}, 36.177$ mmol, 1.0 mol. equiv.) and benzyl bromide $(4.297 \mathrm{~mL}$, $36.177 \mathrm{mmol}, 0.01 \mathrm{~mol}$. equiv) in DMF (50 mL) via general procedure 1 to produce $7.750 \mathrm{~g}$ of $\mathbf{6}$ as a white solid (94\% yield). $R_{\mathrm{f}}=0.52\left(\mathrm{CH}_{2} \mathrm{Cl}_{2} / \mathrm{MeOH}, 20: 1, \mathrm{v} / \mathrm{v}\right) .{ }^{1} \mathrm{H}$ NMR $\left(400.130 \mathrm{MHz}, \mathrm{CDCl}_{3}\right): \delta 5.17(\mathrm{~s}, 1 \mathrm{H}), 5.36(\mathrm{~s}, 2 \mathrm{H}), 7.05$ $\left(\mathrm{dd}, J_{1}=8.0 \mathrm{~Hz}, J_{2}=2.4 \mathrm{~Hz}, 1 \mathrm{H}\right), 7.30-7.45(\mathrm{~m}, 6 \mathrm{H}), 7.56$ (s, $1 \mathrm{H}), 7.66(\mathrm{~d}, J=7.8 \mathrm{~Hz}, 1 \mathrm{H})$.

\section{3. 2. Synthesis of Benzyl 4-Hydroxybenzoate (8)}

Synthesized from 4-hydroxybenzoic acid (9) $(5.000$ g, 36.177 mmol, 1.0 mol. equiv.), $\mathrm{Na}_{2} \mathrm{CO}_{3}(3.837 \mathrm{~g}, 36.177$ mmol, 1.0 mol. equiv.) and benzyl bromide $(4.297 \mathrm{~mL}$, $36.177 \mathrm{mmol}, 1.0$ mol. equiv) in DMF $(50 \mathrm{~mL})$ via general procedure 1 to produce $7.073 \mathrm{~g}$ of $\mathbf{8}$ as a white solid (86\% yield). $R_{\mathrm{f}}=0.46\left(\mathrm{CH}_{2} \mathrm{Cl}_{2} / \mathrm{MeOH}, 20: 1, \mathrm{v} / \mathrm{v}\right) .{ }^{1} \mathrm{H} \mathrm{NMR}$ $\left(400.130 \mathrm{MHz}, \mathrm{CDCl}_{3}\right): \delta 5.34(\mathrm{~s}, 2 \mathrm{H}), 5.58(\mathrm{~s}, 1 \mathrm{H}), 6.86$ $(\mathrm{d}, J=8.7 \mathrm{~Hz}, 2 \mathrm{H}), 7.32-7.45(\mathrm{~m}, 5 \mathrm{H}), 8.00(\mathrm{~d}, J=8.7 \mathrm{~Hz}$, $2 \mathrm{H})$.

\section{3. 3. Synthesis of Benzyl 3-((phenylcarbamoyl) oxy)benzoate (10)}

Synthesized from 6 (3.249 g, $14.235 \mathrm{mmol}, 1.0 \mathrm{~mol}$. equiv.), phenyl isocyanate $(1.547 \mathrm{~mL}, 14.235 \mathrm{mmol}, 1.0$ mol. equiv. ) and 4-DMAP $(0.017 \mathrm{~g}, 0.142 \mathrm{mmol}, 0.01 \mathrm{~mol}$. equiv.) in $\mathrm{CH}_{2} \mathrm{Cl}_{2}(47 \mathrm{~mL})$ via general procedure 2 to produce $4.750 \mathrm{~g}$ of 10 as a white solid (96\% yield). $R_{\mathrm{f}}=0.44$ $\left(\mathrm{CH}_{2} \mathrm{Cl}_{2}\right)$. mp $121-123{ }^{\circ} \mathrm{C}$. IR (ATR): $3319,1707,1544$, $1440,1278,1202,1107,732,692 \mathrm{~cm}^{-1}$. ${ }^{1} \mathrm{H}$ NMR $(400.130$ $\left.\mathrm{MHz}, \mathrm{CDCl}_{3}\right): \delta 5.37(\mathrm{~s}, 2 \mathrm{H}), 6.96($ br. s, $1 \mathrm{H}), 7.13(\mathrm{t}, J=$ $7.3 \mathrm{~Hz}, 1 \mathrm{H}), 7.33-7.49(\mathrm{~m}, 11 \mathrm{H}), 7.89(\mathrm{~s}, 1 \mathrm{H}), 7.97(\mathrm{~d}, J=$ $7.6 \mathrm{~Hz}, 1 \mathrm{H}) .{ }^{13} \mathrm{C}$ NMR $\left(100 \mathrm{MHz}, \mathrm{DMSO}-d_{6}\right): \delta 66.41$, $118.51,122.52,123.06,126.23,127.03,127.97,128.10$, $128.46,128.81,129.98,130.93,135.90,138.39,150.60$, $151.35,164.82$. HRMS (ESI+): $m / z$ calcd for $\mathrm{C}_{21} \mathrm{H}_{18} \mathrm{NO}_{4}$ : 348.12303; found: 348.12410 . Anal. Calcd for $\mathrm{C}_{21} \mathrm{H}_{17} \mathrm{NO}_{4}$ : C, 72.61; H, 4.93; N, 4.03. Found: C, 72.65; H, 4.96; N, 4.00 .

\section{3. 4. Synthesis of Benzyl 3-((ortho- Tolylcarbamoyl)oxy)benzoate (11)}

Synthesized from 6 (3.463 g, $15.172 \mathrm{mmol}, 1.0 \mathrm{~mol}$. equiv.), 2-methylphenyl isocyanate $(1.881 \mathrm{~mL}, 15.172$ mmol, 1.0 mol. equiv.) and 4-DMAP $(0.019 \mathrm{~g}, 0.152$ mmol, 0.01 mol. equiv.) in $\mathrm{CH}_{2} \mathrm{Cl}_{2}(50 \mathrm{~mL})$ via general procedure 2 to produce $5.373 \mathrm{~g}$ of 11 as a white solid (98\% yield). $R_{\mathrm{f}}=0.32\left(\mathrm{CH}_{2} \mathrm{Cl}_{2}\right) \cdot \mathrm{mp} 78-80^{\circ} \mathrm{C}$. IR (ATR): $3273,1712,1531,1289,1270,1232,1189,1069,1022,747$ $\mathrm{cm}^{-1} .{ }^{1} \mathrm{H}$ NMR $\left(400.130 \mathrm{MHz}, \mathrm{CDCl}_{3}\right): \delta 2.34(\mathrm{~s}, 3 \mathrm{H})$, $5.37(\mathrm{~s}, 2 \mathrm{H}), 6.75$ (br. s, $1 \mathrm{H}), 7.08(\mathrm{t}, J=7.3 \mathrm{~Hz}, 1 \mathrm{H}), 7.22$ (t, $J=7.6 \mathrm{~Hz}, 2 \mathrm{H}), 7.33-7.49(\mathrm{~m}, 7 \mathrm{H}), 7.83$ (br. s, $1 \mathrm{H}$ ), $7.89(\mathrm{~s}, 1 \mathrm{H}), 7.96(\mathrm{~d}, J=7.5 \mathrm{~Hz}, 1 \mathrm{H}) .{ }^{13} \mathrm{C} \mathrm{NMR}(100 \mathrm{MHz}$, DMSO- $\left.d_{6}\right): \delta 17.72,66.41,115.66,119.88,120.41,122.42$, $126.08,126.12,126.93,127.91,127.97,128.02,128.09$, $128.45,129.92,130.37,130.90,135.65,135.91,150.92$, $152.33,164.85$. HRMS (ESI+): $m / z$ calcd for $\mathrm{C}_{22} \mathrm{H}_{20} \mathrm{NO}_{4}$ : 362.13868; found: 362.13802 . Anal. Calcd for $\mathrm{C}_{22} \mathrm{H}_{19} \mathrm{NO}_{4}$ : C, 73.12; H, 5.30; N, 3.88. Found: C, 73.11; H, 5.26; N, 3.92 . 


\section{3. 5. Synthesis of Benzyl 3-(((4-Isopropylphenyl) carbamoyl)oxy)benzoate (12)}

Synthesized from 6 (3.242 g, $14.204 \mathrm{mmol}, 1.0 \mathrm{~mol}$. equiv.), 4-isopropylphenyl isocyanate $(2.267 \mathrm{~mL}, 14.204$ mmol, 1.0 mol. equiv. ) and 4-DMAP ( $0.017 \mathrm{~g}, 0.142 \mathrm{mmol}$, 0.01 mol. equiv.) in $\mathrm{CH}_{2} \mathrm{Cl}_{2}(47 \mathrm{~mL})$ via general procedure 2 to produce $5.278 \mathrm{~g}$ of $\mathbf{1 2}$ as a white solid (95\% yield). $R_{\mathrm{f}}=$ $0.45\left(\mathrm{CH}_{2} \mathrm{Cl}_{2}\right)$. mp $99-101^{\circ} \mathrm{C}$. IR (ATR): 3322, 2963, 1710, $1529,1445,1275,1231,1100,741 \mathrm{~cm}^{-1} .{ }^{1} \mathrm{H}$ NMR $(400.130$ $\left.\mathrm{MHz}, \mathrm{CDCl}_{3}\right): \delta 1.24(\mathrm{~d}, J=6.8 \mathrm{~Hz}, 6 \mathrm{H}), 2.84-2.94(\mathrm{~m}$, $1 \mathrm{H}), 5.37$ (s, 2H), 6.92 (br. s, $1 \mathrm{H}), 7.20$ (d, $J=8.3 \mathrm{~Hz}, 2 \mathrm{H})$, 7.33-7.49 (m, 9H), 7.88 (s, 1H), $7.96(\mathrm{~d}, J=7.5 \mathrm{~Hz}, 1 \mathrm{H})$. ${ }^{13} \mathrm{C}$ NMR (100 MHz, DMSO- $\left.d_{6}\right): \delta 23.82,32.74,66.39$, $118.63,122.46,126.13,126.50,126.98,127.95,128.07$, $128.43,129.94,130.88,135.88,136.06,143.13,150.65$, $151.35,164.80$. HRMS (ESI+): $m / z$ calcd for $\mathrm{C}_{24} \mathrm{H}_{24} \mathrm{NO}_{4}$ : 390.16998; found: 390.16931. Anal. Calcd for $\mathrm{C}_{24} \mathrm{H}_{23} \mathrm{NO}_{4}$ : C, 74.02; H, 5.95; N, 3.60. Found: C, 74.05; H, 5.92; N, 3.58.

\section{3. 6. Synthesis of Benzyl 4-((Phenylcarbamoyl) oxy)benzoate (13)}

Synthesized from 8 (3.010 g, $13.187 \mathrm{mmol}, 1.0 \mathrm{~mol}$. equiv.), phenyl isocyanate $(1.433 \mathrm{~mL}, 13.187 \mathrm{mmol}, 1.0$ mol. equiv.) and 4-DMAP (0.016 g, $0.132 \mathrm{mmol}, 0.01 \mathrm{~mol}$. equiv.) in $\mathrm{CH}_{2} \mathrm{Cl}_{2}(44 \mathrm{~mL})$ via general procedure 2 to produce $4.415 \mathrm{~g}$ of 13 as a white solid (96\% yield). $R_{\mathrm{f}}=0.33$ $\left(\mathrm{CH}_{2} \mathrm{Cl}_{2}\right)$. mp 103-105 ${ }^{\circ} \mathrm{C}$. IR (ATR): 3331, 1706, 1543, 1264, 1216, 1102, 1007, 752, $690 \mathrm{~cm}^{-1}$. ${ }^{1} \mathrm{H}$ NMR (400.130 $\mathrm{MHz}, \mathrm{CDCl}_{3}$ ): $\delta 5.34$ (s, 2H), 6.95 (br. s, $\left.1 \mathrm{H}\right), 7.10(\mathrm{t}, J=$ $7.3 \mathrm{~Hz}, 1 \mathrm{H}), 7.24(\mathrm{~d}, J=3.4 \mathrm{~Hz}, 2 \mathrm{H}), 7.30-7.43(\mathrm{~m}, 9 \mathrm{H})$, $8.10(\mathrm{~d}, J=8.6 \mathrm{~Hz}, 2 \mathrm{H}) .{ }^{13} \mathrm{C}$ NMR $\left(100 \mathrm{MHz}, \mathrm{DMSO}-d_{6}\right)$ : $\delta 66.16,115.35,118.15,118.55,122.08,123.13,126.58$, $127.86,128.02,128.43,128.82,130.82,131.50,136.06$, $138.32,150.93,154.43,164.89$. HRMS (ESI+): $\mathrm{m} / z$ calcd for $\mathrm{C}_{21} \mathrm{H}_{18} \mathrm{NO}_{4}$ : 348.12303; found: 348.12249 . Anal. Calcd for $\mathrm{C}_{21} \mathrm{H}_{17} \mathrm{NO}_{4}$ : C, 72.61; H, 4.93; N, 4.03. Found: C, 72.64; $\mathrm{H}, 4.96 ; \mathrm{N}, 4.05$.

\section{3. 7. Synthesis of Benzyl 4-((ortho- Tolylcarbamoyl)oxy)benzoate (14)}

Synthesized from 8 (3.453 g, $15.128 \mathrm{mmol}, 1.0 \mathrm{~mol}$. equiv.), 2-methylphenyl isocyanate $(1.876 \mathrm{~mL}, 15.128$ mmol, 1.0 mol. equiv. $)$ and 4-DMAP $(0.018 \mathrm{~g}, 0.131 \mathrm{mmol}$, 0.01 mol. equiv.) in $\mathrm{CH}_{2} \mathrm{Cl}_{2}(50 \mathrm{~mL})$ via general procedure 2 to produce $4.975 \mathrm{~g}$ of $\mathbf{1 4}$ as a white solid (91\% yield). $R_{\mathrm{f}}=$ $0.27\left(\mathrm{CH}_{2} \mathrm{Cl}_{2}\right)$. mp 87-89 ${ }^{\circ} \mathrm{C}$. IR (ATR): $3264,1705,1531$, $1454,1272,1207,1232,1016,753,696 \mathrm{~cm}^{-1} .{ }^{1} \mathrm{H}$ NMR $\left(400.130 \mathrm{MHz}, \mathrm{CDCl}_{3}\right): \delta 2.34(\mathrm{~s}, 3 \mathrm{H}), 5.37(\mathrm{~s}, 2 \mathrm{H}), 6.76$ (br. s, $1 \mathrm{H}), 7.09$ (t, $J=7.2 \mathrm{~Hz}, 1 \mathrm{H}), 7.22(\mathrm{t}, J=8.1 \mathrm{~Hz}, 2 \mathrm{H})$, $7.29(\mathrm{~d}, J=8.6 \mathrm{~Hz}, 2 \mathrm{H}), 7.33-7.45(\mathrm{~m}, 5 \mathrm{H}), 7.83$ (br. s, $1 \mathrm{H})$, $8.12(\mathrm{~d}, J=8.7 \mathrm{~Hz}, 2 \mathrm{H}) .{ }^{13} \mathrm{C}$ NMR $\left(100 \mathrm{MHz}, \mathrm{DMSO}-d_{6}\right)$ : $\delta 17.70,66.14,115.34,121.98,124.91,126.14,126.40$, $126.98,127.77,127.87,128.03,128.39,128.44,130.39$, $130.79,131.48,135.54,136.06,136.42,151.89,154.74$,
164.90. HRMS (ESI+): $m / z$ calcd for $\mathrm{C}_{22} \mathrm{H}_{20} \mathrm{NO}_{4}$ : 362.13868; found: 362.13803. Anal. Calcd for $\mathrm{C}_{22} \mathrm{H}_{19} \mathrm{NO}_{4}$ : C, 73.12; H, 5.30; N, 3.88. Found: C, 73.16; H, 5.33; N, 3.91.

\section{3. 8. Synthesis of Benzyl 4-((4-Isopropylphenyl) carbamoyl)oxy)benzoate (15)}

Synthesized from 8 (2.988 g, $13.091 \mathrm{mmol}, 1.0 \mathrm{~mol}$. equiv.), 4-isopropylphenyl isocyanate $(2.089 \mathrm{~mL}, 13.091$ mmol, 1.0 mol. equiv. $)$ and 4-DMAP $(0.016 \mathrm{~g}, 0.131 \mathrm{mmol}$, 0.01 mol. equiv.) in $\mathrm{CH}_{2} \mathrm{Cl}_{2}(44 \mathrm{~mL})$ via general procedure 2 to produce $4.960 \mathrm{~g}$ of $\mathbf{1 5}$ as a white solid (97\% yield). $R_{\mathrm{f}}=$ $0.37\left(\mathrm{CH}_{2} \mathrm{Cl}_{2}\right) . \mathrm{mp} 112-114{ }^{\circ} \mathrm{C}$. IR (ATR): 3329, 2962, $1717,1537,1415,1202,1113,1006,831,689 \mathrm{~cm}^{-1} .{ }^{1} \mathrm{H}$ NMR (400.130 MHz, $\left.\mathrm{CDCl}_{3}\right): \delta 1.24(\mathrm{~d}, J=7.0 \mathrm{~Hz}, 6 \mathrm{H})$, 2.84-2.95 (m, 1H), 5.37 (s, 2H), 6.91 (br. s, 1H), 7.21 (d, J $=8.3 \mathrm{~Hz}, 2 \mathrm{H}), 7.27(\mathrm{~d}, J=8.3 \mathrm{~Hz}, 2 \mathrm{H}), 7.33-7.45(\mathrm{~m}, 7 \mathrm{H})$, $8.12(\mathrm{~d}, J=8.6 \mathrm{~Hz}, 2 \mathrm{H}) .{ }^{13} \mathrm{C}$ NMR $\left(100 \mathrm{MHz}, \mathrm{DMSO}-d_{6}\right)$ : $\delta 23.81,32.75,66.13,115.32,118.24,118.67,122.02$, $126.52,127.84,128.01,128.42,131.47,136.05,143.24$, $150.92,154.50,164.88$. HRMS (ESI+): $m / z$ calcd for $\mathrm{C}_{24} \mathrm{H}-$ ${ }_{24} \mathrm{NO}_{4}$ : 390.16998; found: 390.17214. Anal. Calcd for $\mathrm{C}_{24} \mathrm{H}_{23} \mathrm{NO}_{4}$ : C, 74.02; H, 5.95; N, 3.60. Found: C, 73.99; H, $5.99 ; \mathrm{N}, 3.57$.

\section{3. 9. Synthesis of 3-((Phenylcarbamoyl)oxy) benzoic Acid (16)}

Synthesized from 10 (5.163 g, $14.863 \mathrm{mmol}, 1.0$ mol. equiv.) and $10 \% \mathrm{Pd} / \mathrm{C}(0.258 \mathrm{~g}, 5 \%$ mass of 10$)$ in inhibitor-free THF (258 mL) via general procedure 3 to produce $3.720 \mathrm{~g}$ of $\mathbf{1 6}$ as a white solid (96\% yield). $R_{\mathrm{f}}=$ $0.00\left(\mathrm{CH}_{2} \mathrm{Cl}_{2}\right) . \mathrm{mp} 151-153{ }^{\circ} \mathrm{C}$. IR (ATR): 3337, 2564, $1685,1523,1439,1302,1208,1016,754 \mathrm{~cm}^{-1} .{ }^{1} \mathrm{H}$ NMR $\left(400.130 \mathrm{MHz}\right.$, acetone- $\left.d_{6}\right): \delta 7.09(\mathrm{t}, J=7.3 \mathrm{~Hz}, 1 \mathrm{H}), 7.35$ $(\mathrm{t}, J=7.9 \mathrm{~Hz}, 2 \mathrm{H}), 7.50(\mathrm{~d}, J=8.3 \mathrm{~Hz}, 1 \mathrm{H}), 7.57(\mathrm{t}, J=7.9$ $\mathrm{Hz}, 1 \mathrm{H}), 7.63(\mathrm{~d}, J=8.1 \mathrm{~Hz}, 2 \mathrm{H}), 7.86(\mathrm{~s}, 1 \mathrm{H}), 7.93$ (d, $J=$ $7.6 \mathrm{~Hz}, 1 \mathrm{H}), 9.25$ (s, 1H), 11.43 (br. s, $1 \mathrm{H}) .{ }^{13} \mathrm{C}$ NMR $(100$ $\mathrm{MHz}$, acetone- $\left.d_{6}\right): \delta 120.50,124.77,125.13,128.32$, $128.34,130.74,131.35,133.91,140.54,152.98,153.37$, 167.96. HRMS (ESI+): $m / z$ calcd for $\mathrm{C}_{14} \mathrm{H}_{12} \mathrm{NO}_{4}$ : 258.07608; found: 258.07740. UPLC purity, $99 \%$ at 254 $\mathrm{nm}\left(\operatorname{method} \mathrm{A}, t_{\mathrm{R}}=4.130 \mathrm{~min}\right)$.

\section{3. 10. Synthesis of 3-((ortho-Tolylcarbamoyl) oxy)benzoic Acid (17)}

Synthesized from 11 (5.292 g, $14.643 \mathrm{mmol}, 1.0$ mol. equiv. ) and $10 \% \mathrm{Pd} / \mathrm{C}(0.265 \mathrm{~g}, 5 \%$ mass of 11$)$ in inhibitor-free THF (265 mL) via general prodecure 3 to produce $3.902 \mathrm{~g}$ of $\mathbf{1 7}$ as a white solid (98\% yield). $R_{\mathrm{f}}=$ $0.00\left(\mathrm{CH}_{2} \mathrm{Cl}_{2}\right) \cdot \mathrm{mp} 176-178{ }^{\circ} \mathrm{C}$. IR (ATR): 3298, 2565, $1686,1530,1449,1306,1221,1023,942,750 \mathrm{~cm}^{-1} .{ }^{1} \mathrm{H}$ NMR (400.130 MHz, acetone- $\left.d_{6}\right): \delta 2.39(\mathrm{~s}, 3 \mathrm{H}), 7.11(\mathrm{t}, J$ $=7.5 \mathrm{~Hz}, 1 \mathrm{H}), 7.19-7.26(\mathrm{~m}, 2 \mathrm{H}), 7.49(\mathrm{~d}, J=8.3 \mathrm{~Hz}, 1 \mathrm{H})$, $7.56(\mathrm{t}, J=7.9 \mathrm{~Hz}, 1 \mathrm{H}), 7.63(\mathrm{~d}, J=7.9 \mathrm{~Hz}, 1 \mathrm{H}), 7.85$ (s, 
$1 \mathrm{H}), 7.92(\mathrm{~d}, J=7.6 \mathrm{~Hz}, 1 \mathrm{H}), 8.50$ (br. s, $1 \mathrm{H}), 11.32$ (br. s, $1 \mathrm{H}) .{ }^{13} \mathrm{C}$ NMR $\left(100 \mathrm{MHz}, \mathrm{DMSO}-d_{6}\right): \delta 17.76,122.54$, $124.87,125.48,126.18,126.34,129.72,130.43,132.07$, $132.28,135.71,150.85,152.45,166.64$. HRMS (ESI+): $m / z$ calcd for $\mathrm{C}_{15} \mathrm{H}_{14} \mathrm{NO}_{4}$ : 272.09173; found: 272.09150. UPLC purity, $96 \%$ at $254 \mathrm{~nm}\left(\operatorname{method} \mathrm{A}, t_{\mathrm{R}}=4.193 \mathrm{~min}\right)$.

\section{3. 11. Synthesis of 3-(((4-Isopropylphenyl) carbamoyl)oxy)benzoic Acid (18)}

Synthesized from 12 (5.193 g, $13.334 \mathrm{mmol}, 1.0 \mathrm{~mol}$. equiv.) and $10 \% \mathrm{Pd} / \mathrm{C}(0.260 \mathrm{~g}, 5 \%$ mass of 12$)$ in inhibitor-free THF $(260 \mathrm{~mL})$ via general procedure 3 to produce $3.795 \mathrm{~g}$ of $\mathbf{1 8}$ as a white solid (95\% yield). $R_{\mathrm{f}}=0.00\left(\mathrm{CH}_{2}\right.$ $\mathrm{Cl}_{2}$ ). mp $174-176^{\circ} \mathrm{C}$. IR (ATR): 3320, 2961, 2541, 1715, $1682,1538,1450,1274,1225,1017,840 \mathrm{~cm}^{-1} .{ }^{1} \mathrm{H}$ NMR $\left(400.130 \mathrm{MHz}\right.$, acetone- $\left.d_{6}\right): \delta 1.23(\mathrm{~d}, J=7.0 \mathrm{~Hz}, 6 \mathrm{H}), 2.84-$ $2.94(\mathrm{~m}, 1 \mathrm{H}), 7.23(\mathrm{~d}, J=8.4 \mathrm{~Hz}, 2 \mathrm{H}), 7.48(\mathrm{~m}, 4 \mathrm{H}), 7.85(\mathrm{~s}$, $1 \mathrm{H}), 7.92(\mathrm{~d}, J=7.7 \mathrm{~Hz}, 1 \mathrm{H}), 9.18(\mathrm{~s}, 1 \mathrm{H}), 11.41$ (br. s, $1 \mathrm{H})$. ${ }^{13} \mathrm{C}$ NMR (100 MHz, acetone- $\left.d_{6}\right): \delta 25.35,35.19,120.65$, $124.76,128.28,128.31,128.53,131.32,133.82,138.18$, $145.68,153.03,153.39,167.98$. HRMS (ESI+): $\mathrm{m} / z$ calcd for $\mathrm{C}_{17} \mathrm{H}_{18} \mathrm{NO}_{4}$ : 300.12303 ; found: 300.12463 . UPLC purity, $99 \%$ at $254 \mathrm{~nm}$ (method $\left.\mathrm{A}, t_{\mathrm{R}}=4.723 \mathrm{~min}\right)$.

\section{3. 12. Synthesis of 4-((Phenylcarbamoyl)oxy) benzoic Acid (19)}

Synthesized from 13 (4.334 g, $12.477 \mathrm{mmol}, 1.0 \mathrm{~mol}$. equiv.) and $10 \% \mathrm{Pd} / \mathrm{C}(0.217 \mathrm{~g}, 5 \%$ mass of 13$)$ in inhibitor-free THF $(217 \mathrm{~mL})$ via general procedure 3 to produce $3.194 \mathrm{~g}$ of 19 as a white solid (99\% yield). $R_{\mathrm{f}}=0.00\left(\mathrm{CH}_{2}\right.$ $\mathrm{Cl}_{2}$ ). mp $195-197^{\circ} \mathrm{C}$. IR (ATR): $3305,2557,1682,1527$, $1502,1427,1292,1198,1012,752 \mathrm{~cm}^{-1} .{ }^{1} \mathrm{H}$ NMR $(400.130$ $\mathrm{MHz}$, acetone $\left.-d_{6}\right): \delta 7.10(\mathrm{t}, J=7.3 \mathrm{~Hz}, 1 \mathrm{H}), 7.34-7.38(\mathrm{~m}$, $4 \mathrm{H}), 7.63(\mathrm{~d}, J=7.9 \mathrm{~Hz}, 2 \mathrm{H}), 8.10(\mathrm{~d}, J=8.1 \mathrm{~Hz}, 2 \mathrm{H}), 9.28$ (s, $1 \mathrm{H}), 11.10$ (br. s, $1 \mathrm{H}) .{ }^{13} \mathrm{C}$ NMR (100 MHz, DMSO- $\left.d_{6}\right)$ : $\delta 115.07,118.53,121.89,123.14,127.90,128.87,130.82$, 131.49, 138.33, 151.04, 153.98, 166.67. HRMS (ESI+): $m / z$ calcd for $\mathrm{C}_{14} \mathrm{H}_{12} \mathrm{NO}_{4}$ : 258.07608; found: 258.07647. UPLC purity, $98 \%$ at $254 \mathrm{~nm}\left(\operatorname{method} \mathrm{A}, t_{\mathrm{R}}=4.153 \mathrm{~min}\right)$.

\section{3. 13. Synthesis of 4-((ortho-Tolylcarbamoyl) oxy)benzoic Acid (20)}

Synthesized from 14 (4.640 g, $12.839 \mathrm{mmol}, 1.0$ mol. equiv.) and $10 \% \mathrm{Pd} / \mathrm{C}(0.232 \mathrm{~g}, 5 \%$ mass of 14$)$ in inhibitor-free THF $(232 \mathrm{~mL})$ via general procedure 3 to produce $3.384 \mathrm{~g}$ of $\mathbf{2 0}$ as a white solid (97\% yield). $R_{\mathrm{f}}=$ $0.00\left(\mathrm{CH}_{2} \mathrm{Cl}_{2}\right)$. mp $183-185{ }^{\circ} \mathrm{C}$. IR (ATR): 3280, 2555, $1685,1529,1426,1291,1234,1208,1161,748 \mathrm{~cm}^{-1} .{ }^{1} \mathrm{H}$ NMR (400.130 MHz, acetone- $\left.d_{6}\right): \delta 2.39(\mathrm{~s}, 3 \mathrm{H}), 7.11(\mathrm{t}, J$ $=7.5 \mathrm{~Hz}, 1 \mathrm{H}), 7.20-7.27(\mathrm{~m}, 2 \mathrm{H}), 7.36(\mathrm{~d}, J=8.6 \mathrm{~Hz}, 2 \mathrm{H})$, $7.62(\mathrm{~d}, J=7.7 \mathrm{~Hz}, 1 \mathrm{H}), 8.09$ (d, $J=8.6 \mathrm{~Hz}, 2 \mathrm{H}), 8.53$ (br. s, $1 \mathrm{H}), 11.14$ (br. s, $1 \mathrm{H}) .{ }^{13} \mathrm{C}$ NMR (100 MHz, DMSO- $\left.d_{6}\right): \delta$ $17.77,115.14,121.81,124.99,125.56,126.61,127.80$,
$130.46,130.86,131.55,135.63,152.08,154.37,166.75$. HRMS (ESI+): $m / z$ calcd for $\mathrm{C}_{15} \mathrm{H}_{14} \mathrm{NO}_{4}: 272.09173$; found: 272.09436 . UPLC purity, $97 \%$ at $254 \mathrm{~nm}$ (method A, $t_{\mathrm{R}}=4.213 \mathrm{~min}$ ).

\section{3. 14. Synthesis of 4-((4-Isopropylphenyl) carbamoyl)oxy)benzoic Acid (21)}

Synthesized from 15 (4.864 g, $12.489 \mathrm{mmol}, 1.0$ mol. equiv.) and $10 \% \mathrm{Pd} / \mathrm{C}(0.243 \mathrm{~g}, 5 \%$ mass of 15$)$ in inhibitor-free THF $(243 \mathrm{~mL})$ via general procedure 3 to produce $3.720 \mathrm{~g}$ of $\mathbf{2 1}$ as a white solid ( $99 \%$ yield). $R_{\mathrm{f}}=$ $0.00\left(\mathrm{CH}_{2} \mathrm{Cl}_{2}\right)$. mp $196-198{ }^{\circ} \mathrm{C}$. IR (ATR): 3362,2964 , 2547, 1720,1676,1501,1198,1011,828, $758 \mathrm{~cm}^{-1} .{ }^{1} \mathrm{H}$ NMR $\left(400.130 \mathrm{MHz}\right.$, acetone- $\left.d_{6}\right): \delta 1.23(\mathrm{~d}, J=6.8 \mathrm{~Hz}$, $6 \mathrm{H}), 2.84-2.94(\mathrm{~m}, 1 \mathrm{H}), 7.24(\mathrm{~d}, J=8.3 \mathrm{~Hz}, 2 \mathrm{H}), 7.36(\mathrm{~d}, J$ $=8.4 \mathrm{~Hz}, 2 \mathrm{H}), 7.53(\mathrm{~d}, J=8.2 \mathrm{~Hz}, 2 \mathrm{H}), 8.09(\mathrm{~d}, J=8.6 \mathrm{~Hz}$, 2H), 9.19 (s, 1H), 11.19 (br. s, 1H). ${ }^{13} \mathrm{C}$ NMR $(100 \mathrm{MHz}$, DMSO- $\left.d_{6}\right): \delta 23.90,32.83,118.75,121.85,126.60,127.87$, 130.87, 136.08, 143.31, 151.12, 154.14, 166.75. HRMS (ESI+): $m / z$ calcd for $\mathrm{C}_{17} \mathrm{H}_{18} \mathrm{NO}_{4}:$ 300.12303; found: 300.12476 . UPLC purity, $99 \%$ at $254 \mathrm{~nm}\left(\operatorname{method~A,} t_{\mathrm{R}}=\right.$ $4.743 \mathrm{~min})$.

\section{3. 15. Synthesis of 3-((1-(2,3-Dihydro-1H- inden-2-yl)piperidin-3-yl)carbamoyl) phenyl Phenylcarbamate (22)}

To a 50-mL round-bottom flask equipped with a stirring bar, compound $16(0.100 \mathrm{~g}, 0.389 \mathrm{mmol}, 1.0$ equiv) was added followed by $\mathrm{CH}_{2} \mathrm{Cl}_{2}(10 \mathrm{~mL})$. The resulting suspension was stirred and cooled to $0{ }^{\circ} \mathrm{C}$. N,N-Diisopropylethylamine $(0.135 \mathrm{~mL}, 0.778 \mathrm{mmol}, 2.0$ equiv) was added dropwise and the suspension transformed into a solution. TBTU was added and 30 min later solution A (see below) was added dropwise. The reaction mixture was allowed to warm to room temperature and then stirred for 24 hours. During this time a white precipitate formed. The suspension was filtered with suction to produce $0.133 \mathrm{~g}$ of compound 22 as a white solid (75\% yield).

Preparation of solution A: To $25-\mathrm{mL}$ round-bottom flask equipped with a stirring bar, compound 23 (0.112 g, $0.389 \mathrm{mmol}, 1.0$ equiv) was added followed by $\mathrm{CH}_{2} \mathrm{Cl}_{2}(11$ $\mathrm{mL}$ ). The resulting suspension was stirred and cooled to 0 ${ }^{\circ} \mathrm{C}$. $\mathrm{N}, \mathrm{N}$-Diisopropylethylamine $(0.135 \mathrm{~mL}, 0.778 \mathrm{mmol}$, 2.0 equiv) was added dropwise and the suspension transformed into a solution.

Characterization of compound 22: $R_{\mathrm{f}}=0.46(\mathrm{CH}-$ $\left.{ }_{2} \mathrm{Cl}_{2} / \mathrm{MeOH}, 10: 1, \mathrm{v} / \mathrm{v}\right) . \mathrm{mp} 137-139^{\circ} \mathrm{C}$. IR (ATR): 3293, $2953,1716,1629,1538,1226,1211,1021,694 \mathrm{~cm}^{-1} .{ }^{1} \mathrm{H}$ NMR (400.130 MHz, DMSO- $\left.d_{6}\right): \delta 1.33-1.43(\mathrm{~m}, 1 \mathrm{H})$, $1.48-1.60(\mathrm{~m}, 1 \mathrm{H}), 1.73(\mathrm{~d}, J=13.3 \mathrm{~Hz}, 1 \mathrm{H}), 1.83(\mathrm{~d}, J=$ $10.5 \mathrm{~Hz}, 1 \mathrm{H}), 1.96-2.01(\mathrm{~m}, 2 \mathrm{H}), 2.75-2.86(\mathrm{~m}, 3 \mathrm{H}), 2.98-$ $3.05(\mathrm{~m}, 3 \mathrm{H}), 3.20(\mathrm{t}, J=7.7 \mathrm{~Hz}, 1 \mathrm{H}), 3.97$ (br. s, $1 \mathrm{H}), 7.04-$ $7.12(\mathrm{~m}, 3 \mathrm{H}), 7.17-7.18(\mathrm{~m}, 2 \mathrm{H}), 7.33(\mathrm{t}, J=7.7 \mathrm{~Hz}, 2 \mathrm{H})$, $7.39(\mathrm{~d}, J=8.4 \mathrm{~Hz}, 1 \mathrm{H}), 7.50-7.53(\mathrm{~m}, 3 \mathrm{H}), 7.70(\mathrm{~s}, 1 \mathrm{H})$, 
$7.76(\mathrm{~d}, J=7.7 \mathrm{~Hz}, 1 \mathrm{H}), 8.27(\mathrm{~d}, J=7.7 \mathrm{~Hz}, 1 \mathrm{H}), 10.29$ (s, $1 \mathrm{H}) .{ }^{13} \mathrm{C} \mathrm{NMR}\left(100 \mathrm{MHz}, \mathrm{DMSO}-d_{6}\right): \delta 23.75,29.80$, $36.19,36.28,46.47,50.70,55.99,66.07,118.42,120.79$, $123.00,124.17,124.44,124.72,126.19,128.81,129.27$, $135.81,138.42,141.27,150.28,151.54,164.55$. HRMS (ESI+): $m / z$ calcd for $\mathrm{C}_{28} \mathrm{H}_{30} \mathrm{~N}_{3} \mathrm{O}_{3}: 456.22817$; found: 456.22717. UPLC purity, $96 \%$ at $254 \mathrm{~nm}$ (method $\mathrm{A}, t_{\mathrm{R}}=$ $4.420 \mathrm{~min})$.

\section{Results and Discussion}

For the synthesis of 3-((phenylcarbamoyl)oxy)benzoic acid (16), commercially available 3 -hydroxybenzoic acid (7) was treated with benzyl bromide in the presence of $\mathrm{Na}_{2} \mathrm{CO}_{3}$ in $\mathrm{DMF},{ }^{21}$ to provide benzyl 3-hydroxybenzoate (6) in $86 \%$ yield. No further purification of compound 6 was required and the diethyl ether used for the extraction of compound $\mathbf{6}$ was reused for the extraction in the synthesis of benzyl 4-hydroxybenzoate (8) (Scheme 3).

In the second step, compound $\mathbf{6}$ was converted into carbamate 10 with one equivalent of phenyl isocyanate in the presence of a catalytic amount ( 0.01 equivalent) of 4-DMAP in $\mathrm{CH}_{2} \mathrm{Cl}_{2}{ }^{18,19}$ in $96 \%$ yield. Again, no further purification of carbamate $\mathbf{1 0}$ was required. Using one equivalent of phenyl isocyanate, rather than $1.10^{19}$ or 1.20 equivalent, ${ }^{18}$ was found to be an advantage as no over-reaction occurred. As reported previously, excess phenyl isocyanate can undergo an $\mathrm{S}_{\mathrm{E}}$ Ar substitution in the phenyl moiety of the carbamate to produce an amide, which can be difficult to separate from the desired carbamate. ${ }^{19}$ Additionally, $1.0 \mathrm{~mol} \%$ rather than $5 \mathrm{~mol} \%^{18,19}$ of 4 -DMAP was enough to produce the desired carbamate in excellent yield (Scheme 3).

In the third and final step, the benzyl ester $\mathbf{1 0}$ was debenzylated using classic catalytic hydrogenation with gaseous hydrogen and a catalytic amount of $10 \% \mathrm{Pd} / \mathrm{C}^{22}$ (5\% mass of benzyl ester 10) in inhibitor-free THF to produce carboxylic acid 16 in $99 \%$ yield (Scheme 3 ). The hydrogenation was a very clean reaction: no further purification of acid 16 was required and the inhibitor-free THF was reused for the debenzylation of benzyl esters 11-15.

The overall yield for the preparation of compound $\mathbf{1 6}$ from 3-hydroxybenzoic acid (7) using this procedure was $87 \%$ (Table 1). The same procedure was then used to prepare compounds 17-21 from the corresponding hydroxybenzoic acids 7 or 9 via 11-15 (Scheme 3). Overall yields ranged from $76-90 \%$ and are reported in Table 1.

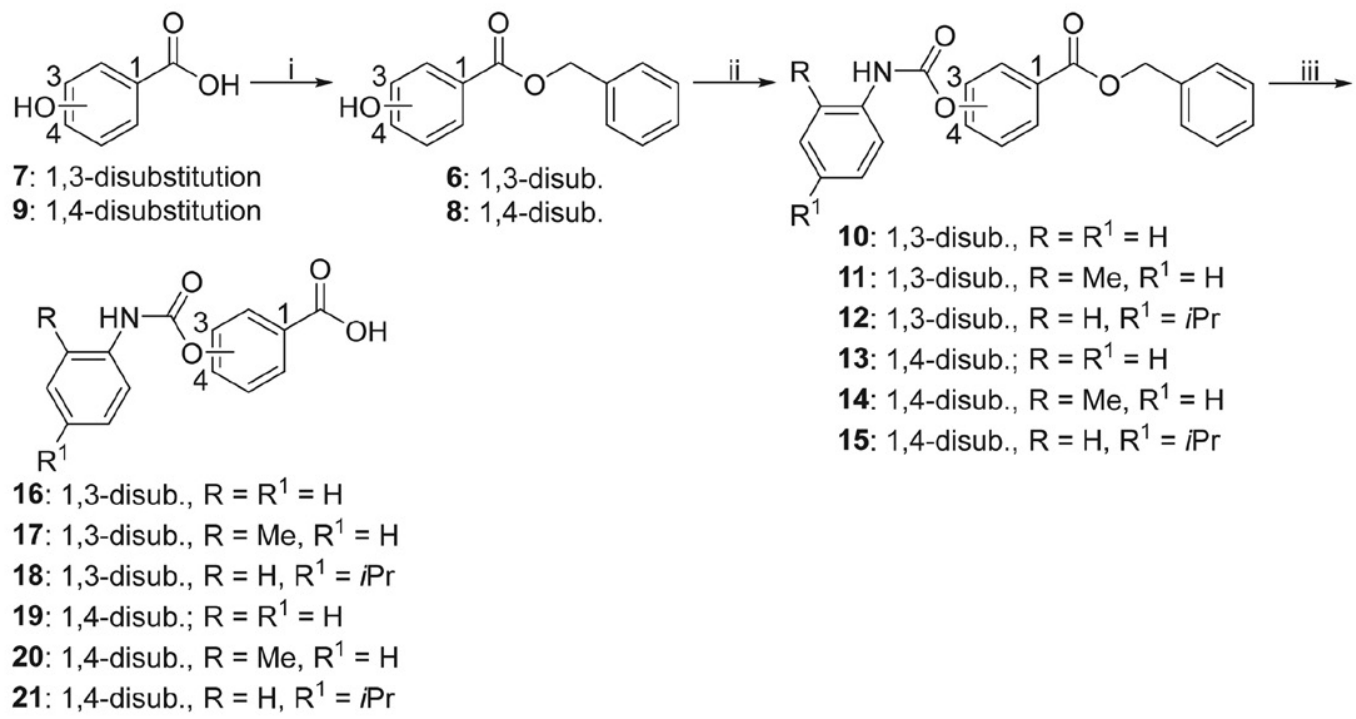

Scheme 3. Reagents and conditions: (i) $\mathrm{PhCH}_{2} \mathrm{Br}, \mathrm{Na}_{2} \mathrm{CO}_{3}, \mathrm{DMF}, \mathrm{rt}, 24 \mathrm{~h}, 94 \%$ (for 8) and 86\% (for 9); (ii) aryl isocyanate, $4-\mathrm{DMAP} \mathrm{CH}_{2} \mathrm{Cl}_{2}, \mathrm{rt}, 24$ h, 91-98\%; (iii) $\mathrm{H}_{2}(\mathrm{~g}), 10 \% \mathrm{Pd} / \mathrm{C}, \mathrm{THF}, \mathrm{rt}, 24$ h, $95-99 \%$.

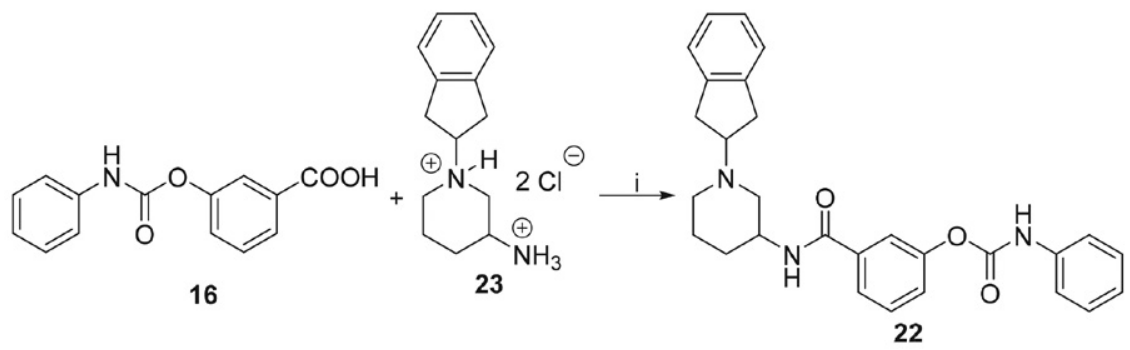

Scheme 4. Reagents and conditions: (i) TBTU, N,N-DIPEA, $\mathrm{CH}_{2} \mathrm{Cl}_{2}, 0^{\circ} \mathrm{C}$ to $\mathrm{rt}, 24 \mathrm{~h}, 75 \%$. 
Table 1. The synthesized 3- and 4-((phenylcarbamoyl)oxy)benzoic acids.

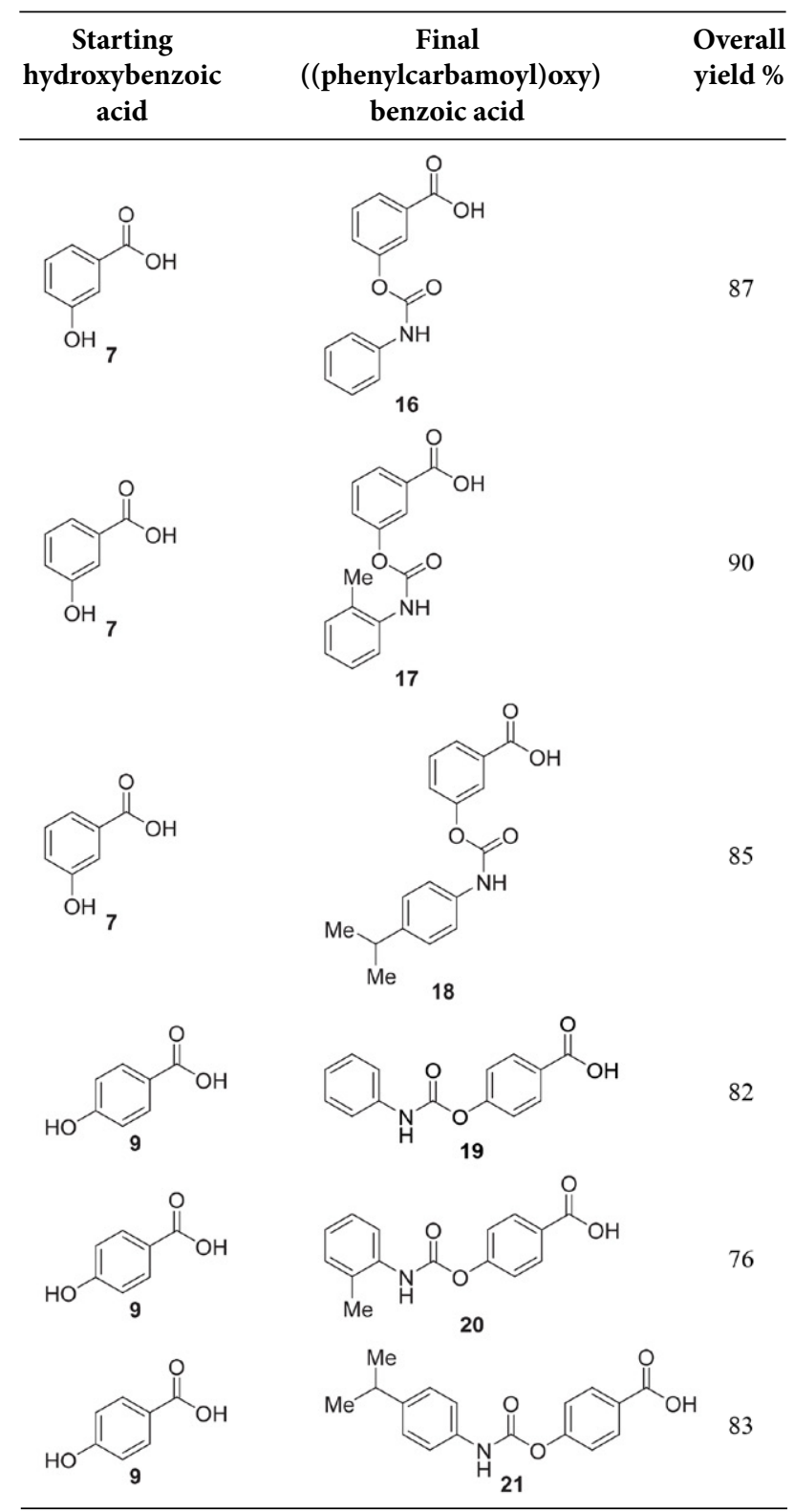

As a proof of concept that the synthesized 3- and 4-((phenylcarbamoyl)oxy)benzoic acids 16-21 can be used in the next reaction to prepare amides, carboxylic acid $\mathbf{1 6}$ was reacted with amine $\mathbf{2 3}$ (which we have previously used to synthesize amide $\mathrm{e}^{13,14}$ and sulfonamide $\mathrm{e}^{14,15} \mathrm{ChE}$ inhibitors), in the presence of coupling reagent TBTU and $\mathrm{N}, \mathrm{N}$-diisopropylethylamine ( $N, N$-DIPEA) in $\mathrm{CH}_{2} \mathrm{Cl}_{2}{ }^{20}$ to produce amide 22 in $75 \%$ yield (Scheme 4 ).

\section{Conclusions}

In summary, we have developed method for the synthesis of previously unreported 3- and 4-((phenylcarbam- oyl)oxy)benzoic acids from commercially available 3- and 4-hydroxybenzoic acids, respectively. The main advantages of our method are the simplicity, as no purification of intermediates or final acids is required, and effectiveness, as the overall yields are very good to excellent (76-90\%). As we have shown, the synthesized carboxylic acids can be converted further, e.g. reacted with amines to produce amides with potential application in drug discovery.

\section{Acknowledgements}

The authors declare that there is no conflict of interest. This work was supported by the Slovenian Research Agency ARRS (grant No. Z1-9195 and core funding P1-0208).

\section{References}

1. C. L. Masters, R. Bateman, K. Blennow, C. C. Rowe, R. A. Sperling, J. L. Cummings, Nat. Rev. Dis. Primers 2015, 1, 15056. DOI: $10.1038 /$ nrdp. 2015.56

2. E. Scherder, in: Aging and dementia: neuropsychology, motor Skills, and pain, 1st ed; VU University Press, Amsterdam, Netherlands, 2011, pp. 9-32.

3. E. Scarpini, P. Schelterns, H. Feldman, Lancet Neurol. 2003, 2, 539-547. DOI:10.1016/S1474-4422(03)00502-7

4. E. K. Perry, B. E. Tomlinson, G. Blessed, K. Bergmann, P. H. Gibson, R.H. Perry, Br. Med. J. 1978, 2, 1457-1459.

DOI:10.1136/bmj.2.6150.1457

5. J. L. Cummings, G. Lee, A. Ritter, M. Sabbagh, K. Zhong, Alzheimers Dement. 2019, 5, 272-293.

DOI:10.1016/j.trci.2019.05.008

6. D. Lecca, M. Bader, D. Tweedie, A. F. Hoffman, Y. J. Jung, S. C. Hsueh, B. J. Hoffer, R. E. Becker, C. G. Pick, C. R. Lupica, N. H. Greig, Neurobiol. Dis. 2019, 130, 104528. DOI:10.1016/j.nbd.2019.104528

7. M. L. Maccecchini, M. Y. Chang, C. Pan, J. Varghese, H. Zetterberg, N. H. Greig, J. Neurol. Neurosurg. Psychiatry. 2012, 83, 894-902. DOI:10.1136/jnnp-2012-302589

8. M. A. Kamal, N. H. Greig, A. S. Alhomida, A. A. Al-Jafari, Biochem. Pharmacol. 2000, 60, 561-70. DOI:10.1016/S0006-2952(00)00330-0

9. N. H. Greig, T. Utsuki, D. K. Ingram, Y. Wang, G. Pepeu, C. Scali, Q. S. Yu, J. Mamczarz, H. W. Holloway, T. Giordano, D. Chen, K. Furukawa, K. Sambamurti, A. Brossi, D. K. Lahiri, Proc. Natl. Acad. Sci. 2005, 102, 17213-17218.

DOI:10.1073/pnas.0508575102

10. M. A. Kamal, X. Qu, Q. S. Yu, D. Tweedie, H. W. Holloway, Y. Li, Y. Tan, N. H. Greig, J. Neural. Transm. 2008, 115, 889-898. DOI:10.1007/s00702-008-0022-y

11. C. Bartolucci, J. Stojan, Q. Yu, N. H. Greig, D. Lamba, Biochem. J. 2012, 444, 269-277. DOI:10.1042/BJ20111675

12. G. L. Patrick, in: An Introduction to Medicinal Chemistry, Oxford Univeristy Press Inc., New York, United States, 2009, pp. 601-603.

13. U. Košak, B. Brus, D. Knez, S. Žakelj, J. Trontelj, A. Pišlar, R. 
Šink, M. Jukić, M. Živin, A. Podkowa, F. Nachon, X. Brazzolotto, J. Stojan, J. Kos, N. Coquelle, K. Sałat, J. P. Colletier, S. Gobec, J. Med. Chem. 2018, 61, 119-139. DOI:10.1021/acs. jmedchem.7b01086

14. U. Košak, D. Knez, N. Coquelle, B. Brusa, A. Pišlar, F. Nachon, X. Brazzolotto, J. Kos, J. P. Colletier, S. Gobec, Bioorg. Med. Chem. 2017, 25, 633-645. DOI:10.1016/j.bmc.2016.11.032

15. U. Košak, B. Brus, D. Knez, R. Šink, S. Žakelj, J. Trontelj, A. Pišlar, J. Šlenc, M. Gobec, M. Živin, L. Tratnjek, M. Perše, K. Sałat, A. Podkowa, B. Filipek, F. Nachon, X. Brazzolotto, A. Więckowska, B. Malawska, J. Stojan, I. Mlinarič Raščan, J. Kos, N. Coquelle, J.-P. Colletier, S. Gobec, Sci. Rep. 2016, 6, 39495. DOI:10.1038/srep39495

16. A. K. Ghosh, M. Brindisi, J. Med. Chem. 2015, 58, 2895-2940.

DOI:10.1021/jm501371s
17. M. T. Leffler, E. J. Matson, J. Am. Chem. Soc. 1948, 70, 34393442. DOI:10.1021/ja01190a065

18. C. Stock, R. Brückner, Synlett 2010, 16, 2429-2434. DOI:10.1055/s-0030-1258552

19. C. Stock, R. Brückner, Adv. Synth. Catal. 2012, 354, 23092330. DOI:10.1002/adsc.201200303

20. S. Balalaie, M. Mahdidoust, R. Eshaghi-Najafabadi, J. Iran. Chem. Soc. 2007, 4, 364-369. DOI:10.1007/BF03245987

21. B. Laursen, M. P. Denieul, T. Skrydstrup, Tetrahedron 2002, 58, 2231-2238. DOI:10.1016/S0040-4020(02)00096-0

22. T. W. Greene, P. G. M. Wuts, in: Protective Groups in Organic Synthesis, John Wiley and Sons, Inc., New York, United States 1999, pp. 416-418. DOI:10.1002/0471220574

\section{Povzetek}

Fenserin, posifen, tolserin in cimserin ter njegovi derivati so eksperimentalne učinkovine za zdravljenje Alzheimerjeve bolezni. Te učinkovine vsebujejo fenil fenilkarbamatno skupino, ki je odgovorna za njihovo delovanje proti Alzheimerjevi bolezni. Razvili smo preprost (trije koraki) in učinkovit (skupni izkoristek 76-90\%) postopek za pripravo 3- in 4-((fenilkarbamoil)oksi)benzojske kisline, ki ju lahko pri reakciji z amini pretvorimo $\mathrm{v}$ amide s fenil fenilkarbamatno skupino. Ti amidi so nove potencialne učinkovine za zdravljenje Alzheimerjevi bolezni. Sintetizirane karboksilne kisline so tako pomembni gradniki, ki se lahko uporabljajo v farmacevtski kemiji in pri odkrivanju zdravilnih učinkovin.

Except when otherwise noted, articles in this journal are published under the terms and conditions of the Creative Commons Attribution 4.0 International License 